\title{
Soliton propagation in optical devices with two-component fields: a comparative study
}

\author{
J. M. Soto-Crespo \\ Instituto de Óptica, Consejo Superior de Investigaciones Científicas, Serrano 121, 28006 Madrid, Spain
}

\author{
Nail Akhmediev and Adrian Ankiewicz
}

Optical Sciences Centre, Institute of Advanced Studies, The Australian National University, Canberra, ACT 0200, Australia

Received March 7, 1994; revised manuscript received November 9, 1994

\begin{abstract}
The problem of solitonlike pulse propagation in optical fiber devices with two-component fields is studied. Two examples, viz., propagation of pulses in birefringent optical fibers and in nonlinear couplers, are considered. It is shown that radiation processes play an essential role in the soliton dynamics in two-component field fiber devices. Radiation influences the transformation of the main pulses in different ways in the two cases considered. The influence of radiation is stronger when the soliton is closer to the point of bifurcation or the separatrix and weaker when it is far from it.
\end{abstract}

\section{INTRODUCTION}

Pulse propagation in two-mode fiber devices can be treated as a problem of the evolution of a dynamical system with an infinite number of degrees of freedom. ${ }^{1}$ The two most frequently studied examples of this kind of problem are pulse propagation in birefringent optical fibers $^{2-4}$ and pulse switching in nonlinear couplers. ${ }^{5-11}$ In each case the full dynamics is quite complicated and cannot be treated analytically in general. The reason is that the systems, even though Hamiltonian, are not integrable. However, they can be considered close to integrable, ${ }^{12}$ and the deviation from the integrable case can be treated as a Hamiltonian perturbation. Hence, if the initial condition is a pulse whose envelope is close to a soliton of the unperturbed system, the pulse propagates without major distortion, ${ }^{4}$ and therefore its propagation can be described by the use of only a few functional parameters. In this way the problem can be reduced to a dynamical system with a finite number of degrees of freedom.

However, the system still has an infinite number of degrees of freedom with an infinite number of potential possibilities of energy exchange between the field components as a result of nonintegrability. One of the consequences is radiation of energy from the leading pulse; this occurs for both the birefringent fiber ${ }^{13}$ and the nonlinear coupler. ${ }^{14}$ Obviously, the radiation influences the behavior of the leading pulse itself. The first analytical study of this influence was presented in Ref. 15. However, the radiation intensity was not calculated in Ref. 15, although the radiation intensity defines the rate of transformation of the main pulse. In this paper we establish qualitative differences between the radiation effects in the two systems.

Different systems governed by two coupled nonlinear Schrödinger equations (NLSE's) can have similar behavior. This is especially true when we consider the cw response of some of these devices. ${ }^{16,17}$ In the particular cases of pulse propagation that we study in this paper, it is radiation emission that makes them qualitatively different. Our comparative study takes into account the existence, in both cases, of a bifurcation point where additional soliton branches start. The new branch is below the symmetric state for a nonlinear coupler, ${ }^{18}$ and it is above the fast solitons for a birefringent fiber. ${ }^{19}$ This difference makes the radiation effect different in these two cases.

In particular, we have found that the radiation effects are stronger when a soliton is closer to the point of bifurcation or the separatrix and weaker when it is far from the point of bifurcation or the separatrix. We study these effects numerically, using the integrated Stokes-parameter formalism. These investigations will assist in the development of analytical approaches to studying the intensity of radiation. The results can be generalized to any other systems described by the same type of equation but with different parameters.

Our results can be useful in studying specific devices like the nonlinear switch. However, in this paper we do not attempt to describe any particular device. We are interested only in the general behavior of the two pulse components along the fiber or the coupler rather than the transmission characteristics of a particular device of a given length. This is a different problem that has been considered in many studies. ${ }^{9}$ Nevertheless, any particular device performance can be predicted, at least qualitatively, using our results. Obviously, the influence of radiation must be included in these studies. For short devices the radiation itself can be small, but it influences the shape of the main pulses. For long devices (in comparison with the beat lengths) the influence of radiation can drastically change the behavior of the main pulses. 


\section{PULSE PROPAGATION IN BIREFRINGENT OPTICAL FIBERS}

Pulse propagation in a birefringent optical fiber can be described in terms of two coupled NLSE's. In a reference frame traveling along the $z$ axis with the common group velocity, this set of equations is of the form ${ }^{4}$

$$
\begin{array}{r}
i U_{\xi}+\beta U+1 / 2 U_{\tau \tau}+\left(|U|^{2}+A|V|^{2}\right) U+(1-A) V^{2} U^{*} \\
=0, \\
i V_{\xi}-\beta V+1 / 2 V_{\tau \tau}+\left(A|U|^{2}+|V|^{2}\right) V+(1-A) U^{2} V^{*} \\
=0,
\end{array}
$$

where $U$ and $V$ are the slowly varying envelopes of the two linearly polarized components of the field along the $x$ and $y$ axes, $\beta$ is half the difference between the propagation constants, $A$ is the normalized ratio of the nonlinear susceptibilities, $\xi$ is the normalized longitudinal coordinate, $\tau$ is the normalized retarded time, and an asterisk denotes a complex conjugate.

The set of Eqs. (1) has at least two integrals (constants of motion), viz., the action (total energy of the pulse)

$$
Q=\int_{-\infty}^{\infty}\left(|U|^{2}+|V|^{2}\right) \mathrm{d} \tau
$$

and the Hamiltonian

$$
\begin{aligned}
H= & \int_{-\infty}^{\infty}\left[1 /{ }_{2}\left(\left|U_{\tau}\right|^{2}+\left|V_{\tau}\right|^{2}\right)\right. \\
& -\beta\left(|U|^{2}-|V|^{2}\right)-1 / 2\left(|U|^{2}+|V|^{2}\right)^{2} \\
& \left.-1 / 2(1-A)\left(U V^{*}-U^{*} V\right)^{2}\right] \mathrm{d} \tau
\end{aligned}
$$

Equations (1) can be written in the canonical form in terms of variational derivatives ${ }^{20}$

$$
i U_{\xi}=\frac{\delta H}{\delta U^{*}}, \quad i V_{\xi}=\frac{\delta H}{\delta V^{*}} .
$$

Equations (3) and (4) define a Hamiltonian dynamical system on an infinite-dimensional phase space of two complex functions $U, V$ that decrease to zero at infinity and can be analyzed by the theory of Hamiltonian systems.

A convenient way to solve the above equations is to use Stokes parameters, ${ }^{4}$ which are defined by

$$
\begin{array}{ll}
s_{0}=|U|^{2}+|V|^{2}, & s_{1}=|U|^{2}-|V|^{2}, \\
s_{2}=U^{*} V+U V^{*}, & s_{3}=-i\left(U^{*} V-U V^{*}\right) .
\end{array}
$$

All these parameters are real functions of $\xi$ and $\tau$. They vary along the fiber, so we call them differential Stokes parameters. Noting that all the fields decay to zero at infinity, we can now write Eqs. (1) by using them:

$$
\begin{aligned}
& \frac{\mathrm{d}}{\mathrm{d} \xi} \int_{-\infty}^{\infty} s_{0} \mathrm{~d} \tau=0, \\
& \frac{\mathrm{d}}{\mathrm{d} \xi} \int_{-\infty}^{\infty} s_{1} \mathrm{~d} \tau=-2(1-A) \int_{-\infty}^{\infty} s_{2} s_{3} \mathrm{~d} \tau, \\
& \frac{\mathrm{d}}{\mathrm{d} \xi} \int_{-\infty}^{\infty} s_{2} \mathrm{~d} \tau=2 \beta \int_{-\infty}^{\infty} s_{3} \mathrm{~d} \tau+2(1-A) \int_{-\infty}^{\infty} s_{1} s_{3} \mathrm{~d} \tau, \\
& \frac{\mathrm{d}}{\mathrm{d} \xi} \int_{-\infty}^{\infty} s_{3} \mathrm{~d} \tau=-2 \beta \int_{-\infty}^{\infty} s_{2} \mathrm{~d} \tau,
\end{aligned}
$$

where the dependence on $\tau$ has been eliminated because of the integration and all the magnitudes are real.
Note that Eqs. (6) are invariant relative to the transformation $(1-A) \rightarrow-(1-A)$ and $s_{i} \rightarrow-s_{i}$. This means that if $1-A$ is negative (i.e., $A>1$ ) we can obtain all solutions of Eqs. (6) from the solutions for positive $1-A$ (i.e., $A<1$ ) by changing the signs of the $s_{i}$. The corresponding solutions can be obtained by the inversion of the solutions and the whole phase space for $s_{i}$ with respect to the origin. This allows us to simplify the analysis by considering only the case $A<1$. This is true, however, only in problems with no loss. The inversion of the Stokes parameters is not followed by the inversion of $\xi$ in Eqs. (6). Hence if losses are involved then the transformation given above does not give new solutions.

\section{TWO USEFUL TRANSFORMATIONS}

If we represent the field components in the form

$$
U=U^{\prime}(\xi, \tau, q) \exp (i \beta \xi), \quad V=V^{\prime}(\xi, \tau, q) \exp (i \beta \xi),
$$

Eqs. (1) become

$$
\begin{aligned}
i U_{\xi}^{\prime}+1 / 2 U_{\tau \tau}^{\prime}+\left(\left|U^{\prime}\right|^{2}+A\left|V^{\prime}\right|^{2}\right) U^{\prime} & +(1-A) V^{\prime 2} U^{\prime *} \\
& \times \exp (-i 4 \beta \xi)=0, \\
i V_{\xi}^{\prime}+1 / 2 V_{\tau \tau}^{\prime}+\left(A\left|U^{\prime}\right|^{2}+\left|V^{\prime}\right|^{2}\right) V^{\prime} & +(1-A) U^{\prime 2} V^{\prime *} \\
& \times \exp (i 4 \beta \xi)=0 .
\end{aligned}
$$

Equations in this form have been used in Ref. 2 to consider pulse propagation in fibers. Obviously, transformation (7) does not change the moduli of the field, but it removes the oscillatory factors from the solution at low intensities. However, these oscillations are the cause of birefringence in the linear limit. Hence, to compare the results with experiment more appropriately, one should use the variables $U$ and $V$.

The Stokes parameters $s_{i}^{\prime \prime}$, defined for the $U^{\prime}$ and $V^{\prime}$ variables, are related to the Stokes parameters $s_{i}$, defined above, by

$$
\begin{aligned}
& s_{0}^{\prime \prime}=\left(\left|U^{\prime}\right|^{2}+\left|V^{\prime}\right|^{2}\right)=s_{0}, \\
& s_{1}^{\prime \prime}=\left(\left|U^{\prime}\right|^{2}-\left|V^{\prime}\right|^{2}\right)=s_{1}, \\
& s_{2}^{\prime \prime}=\left(U^{\prime *} V^{\prime}+U^{\prime} V^{\prime *}\right)=s_{2} \cos (2 \beta \xi)-s_{3} \sin (2 \beta \xi), \\
& s_{3}^{\prime \prime}=\left(U^{\prime *} V^{\prime}-U^{\prime} V^{\prime *}\right)=s_{2} \sin (2 \beta \xi)+s_{3} \cos (2 \beta \xi) .
\end{aligned}
$$

These equations show that the Stokes parameters for the original variables evolve as the Stokes parameters for the new variables but rotate with angular velocity $2 \beta$ around the common $s_{1}$ axis. Hence one can easily transform any solution obtained for one set of variables into a solution for the other set simply by using this rotation.

Another useful transformation is ${ }^{2,21}$

$$
P=U+i V, \quad G=U-i V .
$$

Equations (1) then become

$$
\begin{gathered}
i P_{\xi}+K G+1 / 2 P_{\tau \tau}+\left(\frac{A}{2}|P|^{2}+\frac{2-A}{2}|G|^{2}\right) P=0, \\
i G_{\xi}+K P+1 / 2 G_{\tau \tau}+\left(\frac{2-A}{2}|P|^{2}+\frac{A}{2}|G|^{2}\right) G=0,
\end{gathered}
$$


where $K=\beta$. The variables $P$ and $G$ in this set correspond to the representation of the field in terms of its right and left circularly polarized components, respectively. The main differences between Eqs. (1) and (11) are the appearance in Eqs. (11) of an explicit linear coupling term and the disappearance of the terms containing complex-conjugate variables that coupled the phases of the variables in Eqs. (1). Note that ignoring the last terms in Eqs. (8) would remove this type of coupling (phase coupling), thus completely changing the topology of the solutions. Another important difference is that the cross-phase modulation coefficient has the rescaled value $(2-A) / A$. When $A$ is in the range $0<A<1$, the new coefficient varies in the range $1<(2-A) / A<\infty$.

\section{PULSE PROPAGATION IN NONLINEAR COUPLERS}

Equations (11) also describe pulse propagation in nonlinear couplers, with $P$ and $G$ being the slowly varying pulse amplitudes of the fields in each channel. $K$ now represents the normalized coupling coefficient between the cores. When we set $A=2$, Eqs. (11) describe pulse propagation in dual-core fibers, neglecting cross-phase modulation effects. ${ }^{5}$ In terms of the variables $P$ and $G$ defined by Eqs. (10), the Hamiltonian can be written as

$$
\begin{aligned}
H= & \int_{-\infty}^{\infty}\left[1 / 2\left(\left|P_{\tau}\right|^{2}+\left|G_{\tau}\right|^{2}\right)-1 / 2\left(|P|^{4}+|G|^{4}\right)\right. \\
& \left.-K\left(P G^{*}+P^{*} G\right)\right] \mathrm{d} \tau,
\end{aligned}
$$

and the energy $Q$ is

$$
Q=\int_{-\infty}^{\infty}\left(|P|^{2}+|G|^{2}\right) \mathrm{d} \tau
$$

Their associated differential Stokes parameters can be expressed as

$$
\begin{aligned}
2 s_{0} & =\left(|P|^{2}+|G|^{2}\right)=s_{0}^{\prime}, \\
2 s_{1} & =\left(P G^{*}+P^{*} G\right)=s_{2}^{\prime}, \\
-2 s_{2} & =-i\left(P^{*} G-P G^{*}\right)=s_{3}^{\prime}, \\
-2 s_{3} & =\left(|P|^{2}-|G|^{2}\right)=s_{1}^{\prime} .
\end{aligned}
$$

This represents a permutation of the axes of the Stokes vector and a renormalization. Hence if we use the variables $P$ and $G$ then the analysis will remain unchanged, except for the subscript notation and the signs. Moreover, Eqs. (14) show that the Stokes parameters associated with birefringence in fibers are related to those associated with the nonlinear fiber coupler by a simple permutation of axes and a renormalization.

Equations (14) are remarkable relations because they allow us to consider solitonlike pulse propagation for a variety of physical systems within a unified theory. They show that the same trajectories will represent the solutions of Eqs. (11) when the axes are permuted. Physically, this gives different functions in terms of $U$ and $V$, of course. We should also bear in mind that $A=2 / 3$ for a fiber, whereas $A=2$ for the coupler.

\section{STATIONARY SOLUTIONS AND THEIR STABILITY}

For Hamiltonian systems the stationary solutions play a pivotal role in the dynamics. Let us represent the field components in the form

$$
U=u(\xi, \tau, q) \exp (i q \xi), \quad V=v(\xi, \tau, q) \exp (i q \xi)
$$

where $q$ is the parameter of this family of solutions (nonlinearly induced shift to the wave number) and $u$ and $v$ are real functions of its parameters. Now the equation for finding stationary solutions in the variational formulation can be written as

$$
\delta(H-q Q)=0
$$

This variational formulation of the problem also defines the stability of stationary states. For any fixed $Q$ the stationary state is stable if the corresponding $H$ has a local minimum, with $q$ being a Lagrange multiplier. The differential equations for finding stationary solutions obtained from Eqs. (1) are

$$
\begin{gathered}
1 / 2 u_{\tau \tau}-(q-\beta) u+\left(|u|^{2}+A|v|^{2}\right) u+(1-A) v^{2} u^{*}=0 \\
1 / 2 v_{\tau \tau}-(q+\beta) v+\left(A|u|^{2}+|v|^{2}\right) v+(1-A) u^{2} v^{*}=0
\end{gathered}
$$

where $q$ is the soliton parameter, $2 \pi / q$ is proportional to the soliton period, and the energy of the soliton is proportional to $\sqrt{q}$.

Equations (17) have two simple stationary solutions, viz., a linearly polarized soliton along the slow axis,

$$
u=\frac{\sqrt{2(q-\beta)}}{\cosh (\sqrt{2(q-\beta)} \tau)}, \quad v=0,
$$

and a linearly polarized soliton along the fast axis,

$$
u=0, \quad v=\frac{\sqrt{2(q+\beta)}}{\cosh (\sqrt{2(q+\beta)} \tau)} .
$$

Solution (18) is equivalent to the symmetric soliton state of the coupler,

$$
P=G=\frac{\sqrt{2(q-K)}}{\cosh (\sqrt{2(q-K)} \tau)} \exp (i q \xi),
$$

and solution (19) is equivalent to the antisymmetric soliton state of the coupler,

$$
P=-G=i \frac{\sqrt{2(q+K)}}{\cosh (\sqrt{2(q+K)} \tau)} \exp (i q \xi)
$$

Apart from the solutions considered above, there are also soliton states that are not symmetric. They bifurcate from the symmetric or the fast soliton branches at certain values of $q$ and are asymmetric soliton states $^{18}$ or elliptically polarized soliton states. ${ }^{19}$

If neither birefringence nor coupling is present (i.e., $\beta=K=0$ ), the pulses [Eqs. (20) and (21)] degenerate 
into solitons of a single NLSE:

$$
|P|=|G|=\sqrt{u^{2}+v^{2}}=\frac{\sqrt{2 q}}{\cosh (\sqrt{2 q} \tau)} .
$$

This solution can be linearly polarized along any direction in the $(u, v)$ plane (or can represent two independent solitons propagating in the cores of the coupler).

All the above solutions can be represented on an energydispersion diagram [see Figs. 1(a) and 1(b)]. The energy $Q$ in this diagram is given by Eqs. (2) and (13). Twice the energy of one NLSE soliton [Eq. (22)] is shown as the dashed curve. Intuitively, we can say that the pulse of the perturbed NLSE evolves in such a way that its energy stays in the intervals defined by the strips between the three solid curves in Figs. 1(a) and 1(b), provided that the initial conditions are solitonlike pulses. These strips are then the area of allowed motion for solitonlike pulses. Any given solitonlike pulse can be shown on this diagram by its representative point, which specifies its energy and the average $q$ parameter. The dynamics is different in each of the two stripes in Fig. 1. This notion can be expressed exactly if we represent data on the Hamiltonian-versus-energy diagram [Figs. 1(c) and 1(d)]. For any particular family of stationary solutions, there is a one-to-one correspondence between the $q$ parameter, the energy, and the Hamiltonian, so the curves for stationary solutions have the same topology as that on the energy-dispersion diagrams. Thus there is no significant difference between the two types of diagram. However, the $H-Q$ diagram contains more information. First, the stability criterion discussed above is based on the Hamiltonian. Hence we can expect the lowest of the stationary solutions at any fixed $Q$ to be stable, so we can find stable branches on the $H-Q$ diagrams and transpose them to the energy-dispersion diagram. Second, the values $H$ and $Q$ can be calculated for any initial pulse (in contrast to the $q$ parameter). Hence the representative point on the $H-Q$ diagram corresponding to arbitrary solitonlike initial conditions is rigorously defined. If this point is located inside the strips between the stationary solutions or at least close to them, it will stay in the strip, having some complicated motion, and eventually will converge to the stable stationary solution below the initial point, after emitting some amount of radiation. Although the Hamiltonian and the energy are conserved quantities for the whole solution, they can be calculated separately for the main pulse and for radiation, ${ }^{15}$ so that $H$ and $Q$ for the main pulse can move on the $H-Q$ plane, thus making the transition to stationary stable states visible (see Ref. 15).

These transformations differ in our two cases. The symmetric $(P=G)$ soliton states of the coupler are mathematically equivalent to the slow linearly polarized solitons of the birefringent fiber. In this case (if $A>1$ ) novel (A-type asymmetric) soliton states appear below the lower curves in Figs. 1(a) and 1(c), ${ }^{18}$ and the symmetric pairs lose their stability at the point of bifurcation. ${ }^{22}$ If $A<1$, then bifurcation occurs on the upper curves [fast soliton in Figs. 1(a) and 1(c)]. The upper branch in Fig. 1(a) is for elliptically polarized stationary soliton states. ${ }^{19}$ The main difference between the elliptically polarized stationary soliton states and the A-type asymmetric soliton states is that the curves for these special solutions split off from the lower curves in Figs. 1(b) and 1(d) and from the upper curves in Figs. 1(a) and 1(c). This difference arises because the parameter $A(=2)>1$ for the coupler, whereas $A(=2 / 3)<1$ for the birefringent fiber. Because of these states, new areas of allowed motion for the soliton appear. These are between the fast and the elliptically polarized solutions for the birefringent fibers and between the symmetric and the A-type states for the coupler.

The above difference has important consequences. In the case of Figs. 1(a) and 1(c) the energy and the Hamiltonian for elliptically polarized solutions are higher than the energy and the Hamiltonian for other stationary states. Hence these states may be unstable even if they are elliptical fixed points in an analysis using a reduced number of degrees of freedom (see Section 6) rather than saddle-type points. On the other hand, the asymmetric states in Figs. 1(b) and 1(d) are absolutely stable ${ }^{22}$ (starting from the point with minimum energy) because they have the lowest energy and Hamiltonian of all the stationary solutions.

Another consequence follows: The dashed curve in Fig. 1(b) shows the energy of the soliton of an isolated core. It is close to the A-type state toward the right of the curves. This occurs because, at high energy, the asymmetric A-type state is close (in shape and consequently in functional space) to an individual core soliton. Almost all its energy is in one core. This means that solitons of an individual core will directly excite the A-type states at high energies (higher than at the point of bifurcation). This is the root of the switching phenomenon in the coupler. There is no analog in the birefringent fiber.
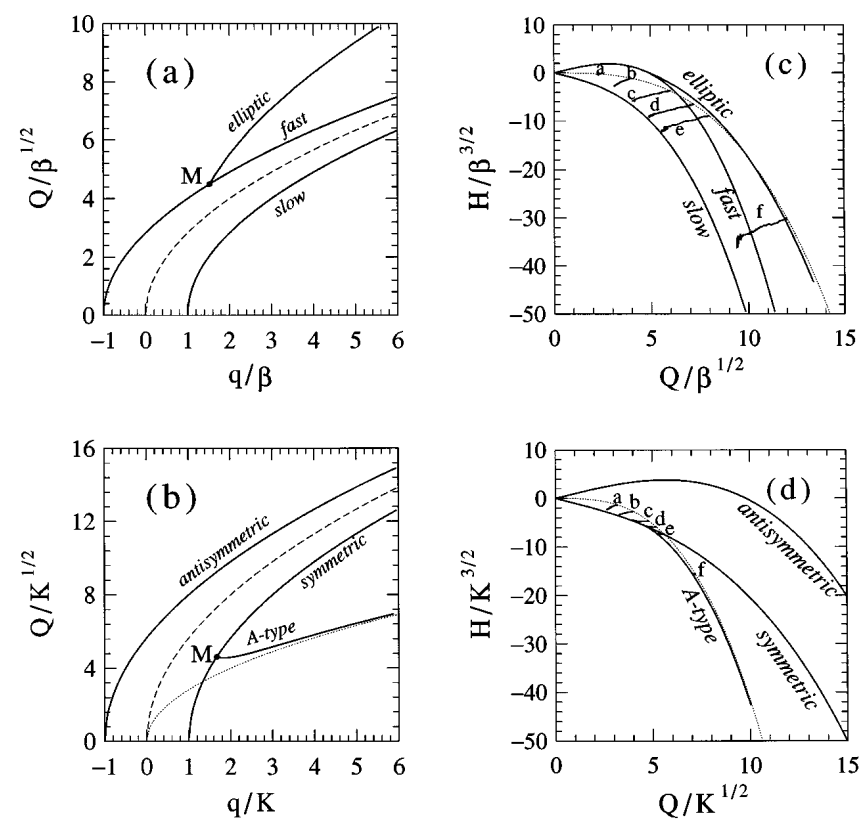

Fig. 1. (a) Energy-dispersion diagram for linearly polarized solitons in birefringent optical fibers. (b) Energy-dispersion diagram for stationary soliton states in nonlinear fiber couplers. (c) Hamiltonian $H$ versus energy $Q$ for linearly polarized solitons in birefringent optical fibers. The dotted curve corresponds to the initial conditions given by Eqs. (33) below. (d) $H$ versus $Q$ for stationary soliton states in nonlinear fiber couplers. The dotted curve represents the initial conditions given by Eqs. (34) below. The trajectories shown by the solid curves correspond to those in Figs. 4 and 5 below. 


\section{APPROXIMATION OF THE AVERAGE PROFILE}

We restrict ourselves to the study of solitonlike pulse dynamics. These pulses form a very special class of solutions, but it is the most important in practice. For solitonlike pulses the shapes of the two components of the pulses should change only slightly on propagation, and the phase chirp across each pulse should be negligible. This idea has been expressed clearly in Ref. 4 (where the whole soliton has the same state of polarization) and can be viewed as a generalization of the soliton phase model ${ }^{23}$ for two-component complex fields. Only the pulse amplitudes should change with distance $\xi$, because of the energy transfer between the two field components.

This is a good approximation to reality for small values of linear birefringence $\beta$ (or coupling $K$ ) relative to the soliton parameter $q$, and it is better when $A$ is close to unity. The former can be expected because, for $K=\beta=0$ and $A=2$, Eqs. (11) separate into two independent integrable equations. Then solitonlike solutions at nonzero $K$ can be considered perturbed solitons of the NLSE. In this approximation the coupling produces only an exchange of energy between cores. On the other hand, $A=1$ corresponds to another integrable case (viz., the Manakov equations ${ }^{24}$ ). Hence the closer $A$ is to unity, the better is our approximation. In this sense the analysis of the birefringent fiber can be more accurate than that of the nonlinear coupler.

To a first approximation, we assume ${ }^{13}$ that the solution is separable in the following way:

$$
u=X(\xi) f(\tau), \quad v=Y(\xi) f(\tau),
$$

where $f(\tau)$ is a real function defining the common profiles and $X(\xi)$ and $Y(\xi)$ are complex amplitudes. Equations (6) become

$$
\begin{aligned}
\frac{\mathrm{d}}{\mathrm{d} \xi} S_{0} & =0, & \frac{\mathrm{d}}{\mathrm{d} \xi} S_{1} & =-2 g S_{2} S_{3}, \\
\frac{\mathrm{d}}{\mathrm{d} \xi} S_{2} & =2 \beta S_{3}+2 g S_{1} S_{3}, & \frac{\mathrm{d}}{\mathrm{d} \xi} S_{3} & =-2 \beta S_{2},
\end{aligned}
$$

where the normalized integrated Stokes parameters $S_{i}=$ $\int_{-\infty}^{\infty} s_{i} \mathrm{~d} \tau / \int_{-\infty}^{\infty} f^{2} \mathrm{~d} \tau$ are given by

$$
\begin{array}{ll}
S_{0}=\left(|X|^{2}+|Y|^{2}\right), & S_{1}=\left(|X|^{2}-|Y|^{2}\right), \\
S_{2}=\left(X^{*} Y+X Y^{*}\right), & S_{3}=-i\left(X^{*} Y-X Y^{*}\right),
\end{array}
$$

and $g$, the nonlinear birefringence coefficient, is defined by

$$
g=(1-A) \frac{\int_{-\infty}^{\infty} f^{4} \mathrm{~d} \tau}{\int_{-\infty}^{\infty} f^{2} \mathrm{~d} \tau} .
$$

Clearly, the value of $g$ depends on $A$ as well as on the soliton shape. On the two edges of the strip of allowed motion the value of $g$ can be calculated exactly. For the upper curves in Fig. $1, g=4 / 3(q+\beta)(1-A)$, and, for the lower curves, $g=4 / 3(q-\beta)(1-A)$. At other points in the area, $g$ is between these values. It is noteworthy that $g$ changes sign at $A=1$.
The set of Eqs. (24) has a constant of motion of

$$
S_{1}^{2}+S_{2}^{2}+S_{3}^{2}=S_{0}^{2},
$$

which is a consequence of energy conservation [Eq. (2)] and indicates that, within the approximation that we are making, the evolution of any solution can be analyzed qualitatively as a trajectory of the Stokes vector $\mathbf{S}$ on the Poincare sphere. The set of Eqs. (24) has a second invariant:

$$
W=\frac{g}{2 \beta} S_{3}^{2}-S_{1},
$$

which is a consequence of the conservation of the Hamiltonian [Eq. (3)], as can easily be verified. In our approximation,

$$
\begin{gathered}
W=\frac{H}{\beta I}+\frac{g S_{0}^{2}}{2 \beta(1-A)}-\frac{\rho S_{0}}{2 \beta}, \\
\rho=\frac{\int_{-\infty}^{\infty} f_{\tau}^{2} \mathrm{~d} \tau}{\int_{-\infty}^{\infty} f^{2} \mathrm{~d} \tau}, \quad I=\int_{-\infty}^{\infty} f^{2} \mathrm{~d} \tau .
\end{gathered}
$$

Different values of $W$, the evolution parameter, correspond to different regimes of soliton propagation (different trajectories on the Poincaré sphere). The convenience of the evolution parameter (in contrast to the Hamiltonian) is that it is constant along the energy-dispersion curves for fast (antisymmetric) and slow (symmetric) solitons in Fig. 1. We can conclude, preliminarily, that a given value of $W$ corresponds to a fixed type of solution as $q$ varies.

Integrated Stokes parameters for the $P$ and $G$ functions can be obtained by permutation of the axes in the same way as that for the differential Stokes parameters:

$$
\begin{array}{ll}
2 S_{0}=S_{0}^{\prime}, & 2 S_{1}=S_{2}^{\prime}, \\
2 S_{2}=-S_{3}^{\prime}, & 2 S_{3}=-S_{1}^{\prime}
\end{array}
$$

All the above equations can easily be written in terms of these parameters. For example, we have, instead of Eqs. (24),

$$
\begin{aligned}
\frac{\mathrm{d}}{\mathrm{d} \xi} S_{0}^{\prime} & =0, & \frac{\mathrm{d}}{\mathrm{d} \xi} S_{2}^{\prime} & =-2 g^{\prime} S_{1}^{\prime} S_{3}^{\prime}, \\
\frac{\mathrm{d}}{\mathrm{d} \xi} S_{3}^{\prime} & =2 K S_{1}^{\prime}+2 g^{\prime} S_{1}^{\prime} S_{2}^{\prime}, & \frac{\mathrm{d}}{\mathrm{d} \xi} S_{1}^{\prime} & =-2 K S_{3}^{\prime},
\end{aligned}
$$

where

$$
g^{\prime}=\frac{1-A}{2} \frac{\int_{-\infty}^{\infty} f^{4} \mathrm{~d} \tau}{\int_{-\infty}^{\infty} f^{2} \mathrm{~d} \tau}=\frac{g}{2} .
$$

The solutions of Eqs. (24) can be written in terms of Jacobi elliptic functions. ${ }^{13,16,17}$ We will concentrate on a qualitative analysis here. There are two stationary (elliptical-type) points when $|g| / \beta>S_{0}$ [Figs. 2(a) and $3(\mathrm{a})]$. They correspond to stationary solutions (18) and (19). There are four stationary points when $|g| / \beta<$ $S_{0} \quad$ [Figs. 2(b) and 3(b)]. Two additional points are analogs of asymmetric soliton states found in Ref. 18 

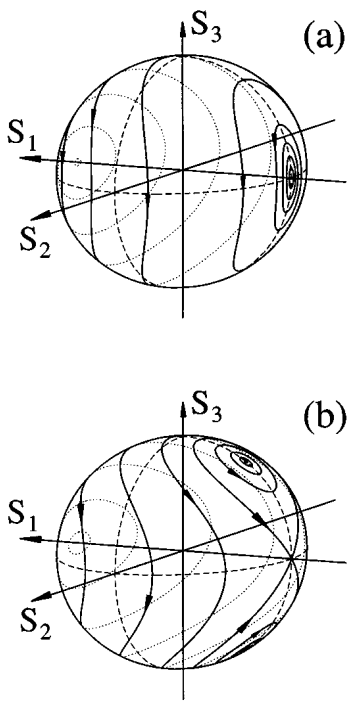

Fig. 2. Trajectories on the Poincare sphere for solutions in the birefringent fiber: (a) $\beta>g S_{0}$, (b) $\beta<g S_{0}$.
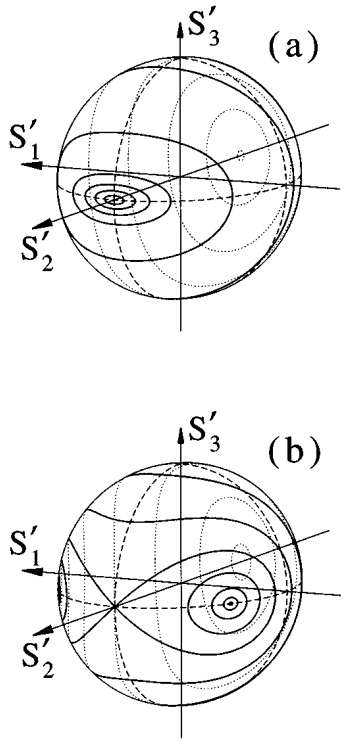

Fig. 3. Trajectories on the Poincaré sphere for the nonlinear coupler: (a) $K>|g| S_{0}$, (b) $K<|g| S_{0}$.

or elliptically polarized soliton states found in Ref. 19. Solutions moving around the stationary elliptical points are periodic. There are two separatrix trajectories connected by a saddle-type point. The full set of trajectories is presented graphically in Fig. 2 .

Trajectories for the $\mathbf{S}^{\prime}$ vector, describing soliton propagation in a nonlinear coupler, are shown in Fig. 3. These trajectories are obtained from Fig. 2 by transformations (30). We recall that the transformations of Eqs. (30) are a consequence of the transformation $g \rightarrow-g$ and $S_{i} \rightarrow-S_{i}$, which, in turn, depends on the inversion symmetry of Eqs. (6). This effectively means that, when the sign of $g$ is changed, the correspondence between the fixed points on the Poincaré sphere and the type of solutions also changes. In the case of the birefringent fiber the elliptical point $\mathbf{S}=\left(S_{0}, 0,0\right)$ corresponds to the lower (slow) branch of the energy-dispersion diagram, whereas, for the nonlinear coupler, the equivalent point $\mathbf{S}^{\prime}=\left(0,-S_{0}^{\prime}, 0\right)$ corresponds to the upper branch (antisymmetric soliton state). The Hamiltonian and the energy decrease when we move over the Poincare sphere from the point corresponding to elliptically polarized solitons to slow solitons in Fig. 2(b), but these values increase when we move from points corresponding to asymmetric states to those for antisymmetric solitons in Fig. 3(b). On account of the radiation effects, this has important consequences when one considers pulse evolution.

The second important difference is related to the value of the parameter $g$. We note that $\left|g^{\prime}\right|$ for the nonlinear coupler is 1.5 times larger than $g$ for the birefringent fiber. Hence the birefringent fiber can be viewed as being closer to the integrable case than is the nonlinear coupler. The result is that at some values of the soliton energy that are slightly below the energy at the bifurcation point [M in Fig. 1(b)], both the symmetric and A-type asymmetric states are stable, and additional unstable A-type states exist. This means that two more fixed points (saddles) can be present on the Poincaré sphere. This is not taken into account in our finite-dimension approximation. Only two (elliptical) fixed points split off from the point corresponding to the symmetric state [see Fig. 3(b)]. This qualitative difference is revealed in our numerical simulations.

\section{RADIATION OF ENERGY FROM THE LEADING PULSE}

Field oscillations of the solitonlike pulse cause it to radiate small-amplitude waves. ${ }^{15}$ Then the values of $Q$ and $H$ for the main pulse must decrease. Hence the representative point of any arbitrary solution always moves down and to the right in Figs. 1(a) and 1(b) and down to the left in Figs. 1(c) and 1(d). A decrease of $Q$ shrinks the Poincaré sphere, but the trajectories on the sphere qualitatively remain the same for fixed $W$. More precisely, the trajectory evolves in such a way that the total energy $Q$, as well as the second invariant $W$, decreases in magnitude. We can take radiation into account by assuming that the values of $Q$ and $W$ decrease adiabatically with $\xi$. Then the solutions behave qualitatively as described above, but the trajectories in Figs. 2 and 3 slowly change from one closed loop to another one with a slightly smaller value of $W$.

On the $H-Q$ diagram (Fig. 1), for a fixed $\beta$ (or $K$ ), we associate a given $W$ and energy $Q$ with a soliton state with predetermined shape and dynamics. At any given $Q$, inside the area of allowed motion, the energy can change from the value for any of the upper curves to the value for any of the lower curves. The second invariant $W$ is equal to $-S_{0}$ for the slow soliton and $+S_{0}$ for the fast soliton. ( $W$ is equal to $-S_{0}$ for the symmetric solution and to $+S_{0}$ for the antisymmetric soliton.) The evolution parameter monotonically decreases from the upper curve to the lower one. However, on the sphere this motion occurs in opposite directions in the two cases of fiber and coupler.

For an infinite length of propagation any initial condition will converge to a slow (A-type) state because this state has the lowest $H$ at a given $Q$. So a trajectory starting at any point on the sphere will tend, on average, to reduce its $W$ value. If this is slow, the trajectories will slide from one closed loop in Figs. 2 and 3 to another one 
with smaller $W$. The decrease in $W$ gradually changes the nature of the solutions and the frequency of oscillations. From our numerical simulations we have found that the rate of this process depends on $\beta /|g|$ and that it is negligible in two cases: for $\beta /|g| \gg 1$ (or $q / \beta \rightarrow 0$ ) and $\beta /|g| \ll 1$ (or $q / \beta \rightarrow \infty$ ). It becomes faster when the representative point is close to the point of bifurcation or crosses the separatrix trajectories.

According to the Kolmogorov-Arnol'd-Moser theorem (which we assume is applicable to systems with an infinite number of degrees of freedom), a finite fraction of the phase-space trajectories is regular, that is, associated with integrals of motion. These paths can be described by adiabatic changes in the constants of motion. They must start far from the points of bifurcation or the separatrices. Irregular trajectories are associated with representative points close to the points of bifurcations or the separatrices. Our numerical simulations confirm this. When the representative point is far from the point of bifurcation or the separatrix, trajectories are regular and radiation is small. The slow decrease of $Q$ and $W$ is connected with the slow reshaping of the average shape of the pulse. On the other hand, when the representative point is close to the point of bifurcation or the separatrix, radiation is large and the trajectory does not follow those for integrable cases.

\section{NUMERICAL SIMULATIONS}

We have numerically solved Eqs. (17) [or equivalently Eqs. (11)] by using a split-step method. ${ }^{13}$ In all the simulations we used the value $A=2 / 3$ for the birefringent fiber and $A=2$ for the coupler. The Stokes parameters are calculated from the values of $u$ and $v$ (or $P$ and $G$ ). First, we study soliton propagation in birefringent fibers. Figure 4 shows the evolution of the Stokes parameters for an input:

$$
u=\frac{Q}{4} \operatorname{sech}\left(\frac{Q}{4} \tau\right), \quad v=-i u \text {. }
$$

Depending on the initial energy, these initial conditions excite the solution with a representative point close to the highest branch at a given energy [see Fig. 1(c)]. Six different cases are shown in Fig. 4. In all six cases the trajectory spirals. It makes many loops parallel to those defined by the approximation of the average profile. $Q$ and $H$ of the main pulse continuously decrease. We have superimposed the changes of these integrals upon Fig. 1(c), using the same labels. In Figs. $4(\mathrm{~d})-4(\mathrm{f})$ the trajectory passes the separatrix and starts to oscillate beyond it. Radiation is maximal when the trajectory passes the separatrix close to the point of bifurcation [see Figs. 4(d) and $4(\mathrm{e})]$. In the case of Fig. $4(\mathrm{f})$ radiation decreases. The trajectory ends up converging to the slow soliton, where $Q$ and $H$ are minimal. Radiation decreases when the trajectory approaches the slow soliton. Note the decrease in the radius of the sphere during the evolution.

We have performed various other simulations for the case of the birefringent fiber. All of them are qualitatively in agreement with the above theory. The only requirement is that the initial condition must be a solitonlike pulse, i.e., close to one of the stationary or other solitonlike solutions of Eqs. (1). Pulses of arbitrary shape will first transform into one or several solitonlike pulses during the evolution while emitting large amounts of radiation in the initial stages. The evolution of each subpulse can be described by the above theory. All the trajectories gradually converge to the slow soliton, with the cases shown in Fig. 4 being typical.

We will now concentrate on the nonlinear coupler. The trajectories can be more complicated in this device for several reasons. The main difference from the birefringent fiber is that A-type asymmetric state has minimal $H$ at given $Q$, whereas the antisymmetric state has maximal $H$ at given $Q$. Radiation causes transitions from one closed loop to another, in reverse of what occurs in the birefringent fiber. Thus, depending on the initial condition, the trajectory can converge to a symmetric state or an asymmetric A-type state. Figure 5 shows six examples of evolution of the Stokes parameter for the nonlinear coupler with $K=1$. The initial condition is a soliton of the unperturbed system, with different values of $Q$ being chosen, i.e.,

$$
P=0, \quad G=\frac{Q}{2} \operatorname{sech}\left(\frac{Q}{2} \tau\right) .
$$

On the Poincaré sphere this initial condition corresponds to the point $\mathbf{S}^{\prime}=\left(-S_{0}^{\prime}, 0,0\right)$. At small energies $(Q=3.2$ and $Q=4.0$ ) the trajectory rotates around the axis $S_{2}^{\prime}$. Radiation is relatively large during the first half-period of
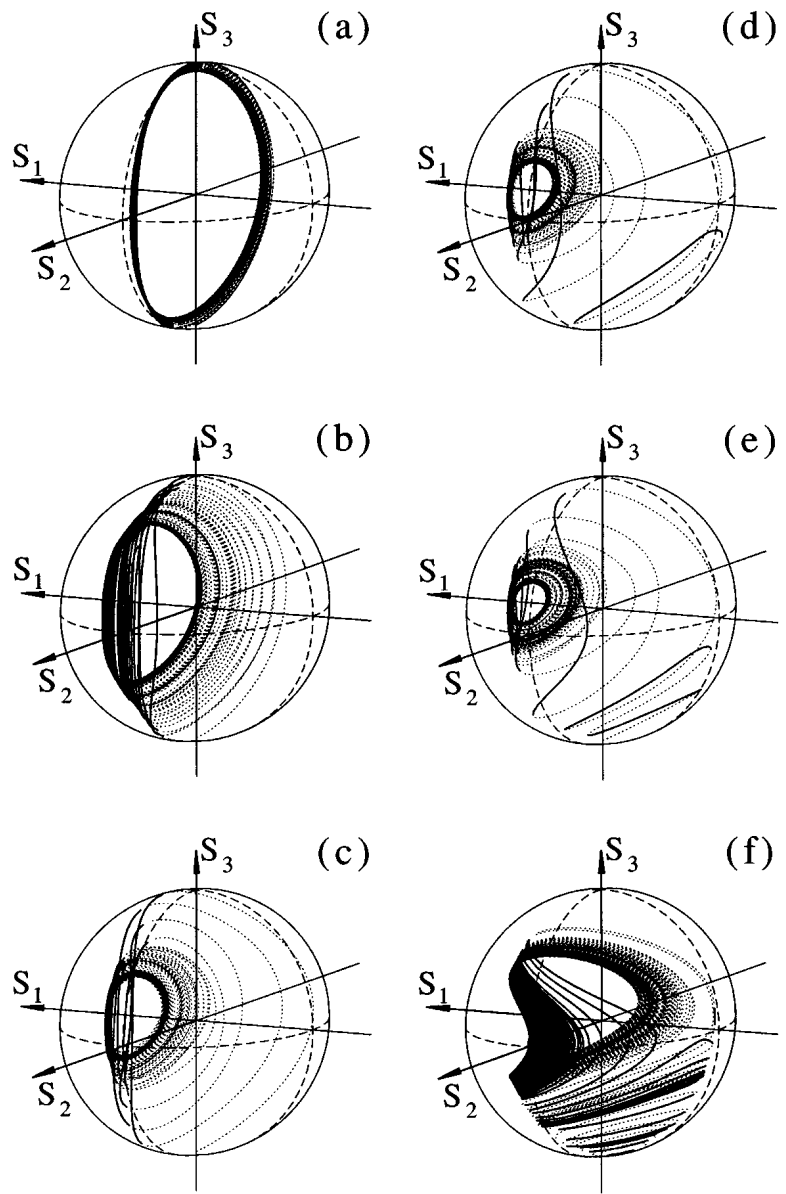

Fig. 4. Evolution of the integrated Stokes parameters for pulses in a birefringent fiber. The initial condition is given by Eqs. (33) with $Q=$ (a) 2.4, (b) 4.0, (c) 6.0, (d) 7.2, (e) 8.0, and (f) 12.0 . 

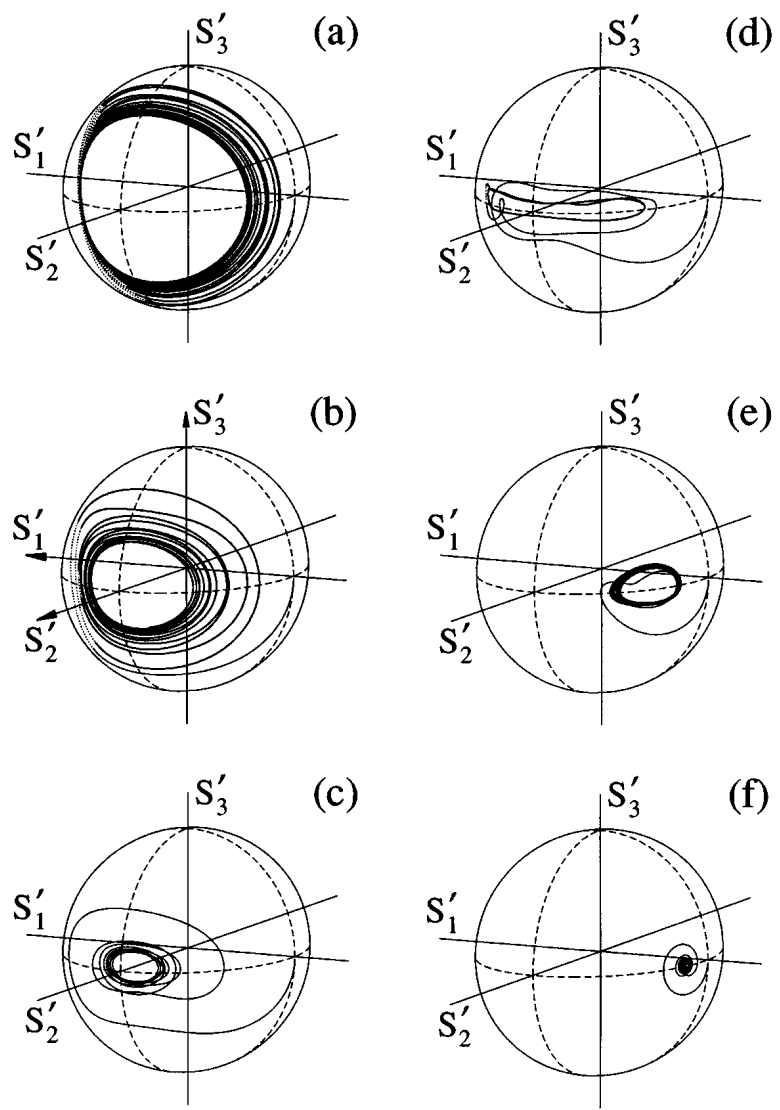

Fig. 5. Evolution of the integrated Stokes parameters for pulses in a nonlinear coupler. The initial condition is a soliton of an individual core as given by Eqs. (34) with $Q=$ (a) 3.2 , (b) 4.0, (c) 4.8 , (d) 5.2 , (e) 5.6 and (f) 7.2 . All the simulations have $K=1$.

this rotation but then becomes smaller. This shows that the initial condition, even if it is in the form of Eqs. (34), is not an exact solution. It must adjust to a solitonlike pulse before it follows the solution defined by our simple analytical approach. At higher values of $Q$ (e.g., $Q=4.8$ ), when the initial condition is closer to the bifurcation point [see Fig. 1(d)], the radiation at initial states of the process is greater, and then the trajectory slowly converges to a stationary symmetric state [Fig. 5(c)].

Initial radiation becomes even larger when $Q=5.2$. However, after the relaxation we have a unique situation, and the behavior is qualitatively out of the domain of our simple model. There are six singular points on the Poincaré sphere rather than just four. Four of them correspond to A-type states (two stable and two unstable). The trajectory converges quickly to a closed loop enclosing four A-type states and a symmetric state [Fig. 5(d)]. This can happen because the energy-dispersion curve for A-type states has a minimum, and so three soliton states can have the same energy. The appearance of the two additional unstable A-type states at the same energy is not predicted by our simple approximation of the average profile. This energy, $Q=5.2$, is approximately $10 \%$ above the bifurcation point energy $\left(Q_{M} \approx 4.62\right)$. The excess energy is radiated during the first part of the process. If the energy of the initial soliton is higher, e.g., $Q=5.6$, then the solution converges to an A-type state [Fig. 5(e)]. At higher values of energy (e.g., $Q=7.2$ ) the ellipticaltype point on the sphere is closer to the initial point
$\mathbf{S}^{\prime}=\left(-S_{0}^{\prime}, 0,0\right)$, and convergence to an A-type state occurs very quickly [Fig. $5(\mathrm{f})$ ].

To show the existence of additional fixed points on the sphere at energies slightly higher than that at the point of minimum (but lower than that at the point of bifurcation), we took an unstable A-type asymmetric state and propagated it, after having added a small perturbation. The results are shown in Fig. 6. The initial A-type state is associated with $q=1$ for a coupler with $K=0.59$. Figure 6(a) shows two periodic orbits inside a complicated set of three-petal separatrices connecting two saddle-type points. The two orbits are produced when two different perturbations are added to the same initial unstable A-type state. One of them (the one at the right) rotates around a stable A-type state, and the other rotates around a symmetric state. The periodic orbit enclosing the full set of three-petal separatrices is reached in the case exhibited in Fig. 5(d). Figure 6(a) shows that additional saddle fixed points appear to the point $\mathbf{S}^{\prime}=\left(0, S_{0}^{\prime}, 0\right)$. Point $\mathbf{S}^{\prime}=\left(0, S_{0}^{\prime}, 0\right)$ itself is still an elliptical point at these values of energy (below the bifurcation point). Figure 6(b) shows the energy distribution between the cores corresponding to the two periodic orbits shown in Fig. 6(a). The solid curves show the oscillations around the symmetric state, and the dashed curves indicate the oscillations around the A-type state. No appreciable energy is radiated because the two stationary points are very close in Hilbert space. The difference in their $H$ values is too small to be seen on the scale of Fig. 1(d).

In the case of Figs. 1(e) and 1(f) the initial conditions are chosen in such a way that the trajectory follows the separatrix in the initial stages of the process. Hence initially the radiation is still large. However, after a half-beat length, the trajectory starts to spiral around the stationary A-type state, and the amount of radiation becomes noticeably smaller.
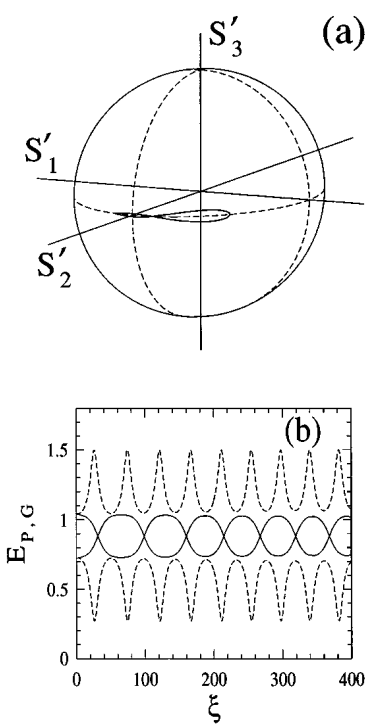

Fig. 6. (a) Evolution of the integrated Stokes parameters for a soliton pair in a nonlinear coupler with $K=0.59$. The initial condition is the unstable A-type asymmetric soliton state with $q=1$, plus a small perturbation. The perturbation produces oscillations around a symmetric or a stable asymmetric state. (b) Evolution of the component energies in each core: $E_{P, G}=\int_{-\infty}^{\infty}|P, G|^{2} \mathrm{~d} \tau$. 

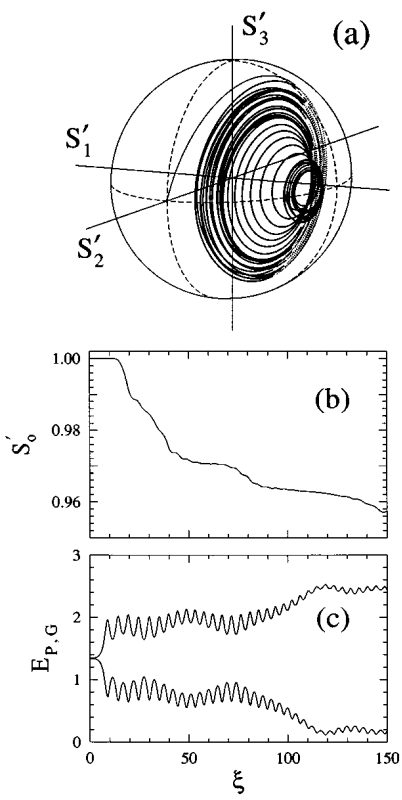

Fig. 7. (a) Evolution of the integrated Stokes parameters for a soliton pair in a nonlinear coupler having $K=0.1$. The initial condition is a symmetric soliton state with $q=1$, plus a small perturbation. (b) Evolution of $S_{0}^{\prime}$ (total energy in the pulse). (c) Evolution of the component energies in each core: $E_{P, G}=\int_{-\infty}^{\infty}|P, G|^{2} \mathrm{~d} \tau$

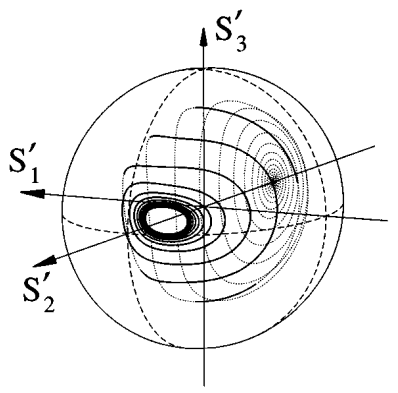

Fig. 8. Evolution of the integrated Stokes parameters for a soliton pair in a nonlinear coupler with $K=1$. The initial condition is the antisymmetric soliton state with $q=0.6$, plus a small perturbation.

To show qualitatively the influence of radiation on the pulse evolution, we also considered two examples with initial conditions different from Eqs. (34). An example of a trajectory that starts from the symmetric state $\mathbf{S}^{\prime}=\left(0, S_{0}^{\prime}, 0\right)$ with a small even perturbation is shown in Fig. 7(a). The values $K=0.1$ and $q=1$ are used in this case. The point $\mathbf{S}^{\prime}=\left(0, S_{0}^{\prime}, 0\right)$, corresponding to a symmetric state, is then a saddle-type point. Hence the trajectory moves away from this point exponentially along the separatrix, but, because of radiation, it slides from the separatrix to a closed periodic orbit. The stationary points corresponding to A-type states are then near the points $\mathbf{S}^{\prime}=\left( \pm S_{0}^{\prime}, 0,0\right)$. The trajectory is a spiral that ends up close to an A-type state. Radiation is relatively large initially, moving the trajectory inside the separatrix, but becomes smaller in the rest part of the pulse evolution. The evolution of $S_{0}^{\prime}$, normalized to its initial value, is shown in Fig. 7(b). Figure 7(c) shows the distribution of energy between cores.

An example of a trajectory that starts from an antisymmetric state $\mathbf{S}^{\prime}=\left(0,-S_{0}^{\prime}, 0\right)$ (with a small perturba- tion) is shown in Fig. 8. The initial state is an elliptical fixed point, but the trajectory spirals out from this point because of radiation and gradually converges to a symmetric state emitting radiation. The whole solution behaves qualitatively, in accordance with our simple model. For higher values of initial energy of the antisymmetric state the behavior is more complicated on account of the existence of odd perturbation ${ }^{22}$ with growth rates much larger than those of any even perturbation. The whole solution then becomes more involved and cannot be described in terms of our simple approximation.

\section{CONCLUSION}

We studied the problem of solitonlike pulse propagation in optical fiber devices with two-component fields. The propagation of pulses in birefringent optical fibers and in nonlinear couplers is considered. It is shown that radiation processes play an essential role in the soliton dynamics in these devices. The influence of radiation is stronger when the soliton is closer to the point of bifurcation or the separatrix and weaker when it is far from it.

\section{ACKNOWLEDGMENTS}

We thank the Australian Photonics Cooperative Research Centre. The work of J. M. Soto-Crespo was supported by the Comisión Interministerial de Ciencia y Technología under contract TIC91-0361. N. Akhmediev thanks A. W. Snyder for stimulating discussions.

\section{REFERENCES}

1. M. Romagnoli, S. Trillo, and S. Wabnitz, "Soliton switching in nonlinear couplers," Opt. Quantum Electron. 24, S1237-S1267 (1992).

2. K. J. Blow, N. J. Doran, and D. Wood, "Polarization instabilities for solitons in birefringent fibers," Opt. Lett. 12, 202-204 (1987).

3. C. R. Menyuk, "Pulse propagation in an elliptically birefringent Kerr medium," IEEE J. Quantum Electron. 25, 2674-2682 (1989).

4. S. G. Evangelides, L. F. Mollenauer, J. P. Gordon, and N. S. Bergano, "Polarization multiplexing with solitons," J. Lightwave Technol. 10, 28-35 (1992).

5. S. Trillo, S. Wabnitz, E. M. Wright, and G. I. Stegeman, "Soliton switching in fiber nonlinear directional couplers," Opt. Lett. 13, 672-674 (1988).

6. M. V. Tratnik and J. E. Sipe, "Nonlinear polarization dynamics. I. The single-pulse equations," Phys. Rev. A 35, 2965-2975 (1987)

7. F. Kh. Abdullaev, R. M. Abrarov, and S. A. Darmanyan, "Dynamics of solitons in coupled optical fibers," Opt. Lett. 14, 131-133 (1989).

8. J. M. Soto-Crespo and E. M. Wright, "All-optical switching of solitons in two- and three-core nonlinear fiber couplers," J. Appl. Phys. 70, 7240-7243 (1991).

9. P. A. Bélanger and C. Paré, "Soliton switching and energy coupling in two-mode fibers: analytical results," Phys. Rev. A 41, 5254-5256 (1990).

10. P. L. Chu, G. D. Peng, and B. Malomed, "Analytical solution to soliton switching in nonlinear twin-core fibers," Opt. Lett. 18, 328-330 (1993).

11. E. M. Wright, G. I. Stegeman, and S. Wabnitz, "Solitarywave decay and symmetry-breaking instabilities in twomode fibers," Phys. Rev. A 40, 4455-4466 (1989).

12. C. R. Menyuk, "Soliton robustness in optical fibers," J. Opt. Soc. Am. B 10, 1585-1591 (1993).

13. N. N. Akhmediev and J. M. Soto-Crespo, "Dynamics of solitonlike pulse propagation in birefringent optical fibers," Phys. Rev. E 49, 5742-5754 (1994). 
14. N. N. Akhmediev and J. M. Soto-Crespo, "Propagation dynamics of ultrashort pulses in nonlinear fiber couplers," Phys. Rev. E 49, 4519-4529 (1994).

15. A. V. Buryak and N. N. Akhmediev, "Influence of radiation on soliton dynamics in nonlinear fibre couplers," Opt. Commun. 110, 287-292 (1994).

16. G. Gregory and S. Wabnitz, "New exact solutions and bifurcations in the spatial distribution of polarization in thirdorder nonlinear optical interactions," Phys. Rev. Lett. 56, 600-603 (1986).

17. B. Daino, G. Gergory, and S. Wabnitz, "Stability analysis of nonlinear coherent coupling," J. Appl. phys. 58, 4512-4514 (1985).

18. N. N. Akhmediev and A. Ankiewicz, "Novel soliton states and bifurcation phenomena in nonlinear fiber couplers," Phys. Rev. Lett. 70, 2395-2398 (1993).
19. N. N. Akhmediev, A. V. Buryak, and J. M. Soto-Crespo, "Elliptically polarized solitons in birefringent optical fibers," Opt. Commun. 112, 278-282 (1994).

20. L. D. Faddeev and L. A. Takhtajan, Hamiltonian Methods in the Theory of Solitons (Springer-Verlag, Berlin, 1987).

21. H. G. Winful, "Self-induced polarization changes in birefringent optical fibers," Appl. Phys. Lett. 47, 213-215 (1985).

22. J. M. Soto-Crespo and N. N. Akhmediev, "Stability of soliton states in a nonlinear fiber coupler," Phys. Rev. E 48, 4710-4715 (1993).

23. K. J. Blow, N. J. Doran, and S. J. D. Phoenix, "The soliton phase," Opt. Commun. 88, 137-140 (1992).

24. S. V. Manakov, "On the theory of two-dimensional stationary self-focusing of electromagnetic waves," Zh. Eksp. Teor. Fiz. 65, 505-516 (1973) [Sov. Phys. JETP 38, 248-253 (1974)]. 